\title{
Diagnosis of Diabetic Retinopathy by Blood Vessel Segmentation and Exudate Detection
}

\author{
Lekshmi Shyam \\ Dept. of Electronics and Communication, \\ SCT College Of Engineering, Pappanamcode, \\ Thiruvananthapuram-18 \\ Kumar G.S \\ Assistant Professor, \\ Dept. of Electronics and Communication, \\ SCT College of Engineering, Pappanamcode, \\ Thiruvananthapuram-18
}

\begin{abstract}
Blindness is a growing problem all over the world. The major diseases that lead to blindness are glaucoma and diabetic retinopathy. Even though these diseases lead to irreversible blindness, it can be controlled to certain extent if detected earlier. Diabetic Retinopathy can be detected by the presence of exudates. The paper proposes a method for vessel segmentation based on high pass filtering and morphological reconstruction methods. The blood vessel segmentation is an important task in Diabetic Retinopathy detection. Optic disc in the fundus image is detected by Hough transform. After the segmentation the vessels and optic disc are removed from the original image. Diabetic Retinopathy is characterized by the presence of exudates. The exudates are detected by means of imtool operator in the matlab. The simulations are performed on matlab 2011 and the data are collected from DIARETDB1 and HRF databases.
\end{abstract}

Keywords-Exudates, High pass filtering, Morphological reconstruction, Hough Transform, Imtool operator

\section{I.INTRODUCTION}

Blindness is a growing problem all over the world. The main causes of blindness are the diseases Glaucoma and Diabetic Retinopathy. Glaucoma is caused due to an increase in the intraocular pressure. Diabetic Retinopathy occurs due to the complexity of Diabetes. In diabetic retinopathy, fluid from the blood leaks and gets deposited in the retinal regions. These occur in the form of yellow spots called exudates. Thus Diabetic Retinopathy can be detected by the presence of exudates. For effective treatment, the disease has to be detected earlier. Diabetic Retinopathy leads to damage to the blood vessels and also formation of lesions. Another complexity of the disease is that new abnormal blood vessels grow in the retina, known as revascularization. These abnormalities lead to severe visual problems. If the exudates extend to the macular region it will lead to severe vision loss. For the detection of exudates, first the blood vessels in the retinal regions are to be segmented. After the segmentation of blood vessels, optic disc is to be segmented. The blood vessels are to be removed to detect the presence of exudates. The segmentation of blood vessels is carried out on fundus images. The fundus images are captured by means of a fundus camera. A fundus camera is a specialized low power microscope with an attached camera. The proposed method employees high pass filtering and morphological reconstruction for blood vessel segmentation. The optic disc detection is performed by Hough transform.

Several approaches have been presented earlier for the detection of diabetic retinopathy. The exudates are detected by blob detection and feature extraction as in [1]. The optic disc center is found by back through radii method. After detecting the optic disc, it is blackened and removed. Binary thresholding method is used to identify the presence of exudates. Probabilistic Neural network (PNN), Bayesian Classification and Support vector machine (SVM) are described and their performances are compared to diagnose Diabetic Retinopathy [2]. The classifier 
makes use of features like blood vessels, haemmoraghes of NPDR image and exudates of PDR image. Detection of exudates and non-exudate detection is performed by means of BPN model at pixel level [3]. Decision tree and GACFS method are used as input to the BPN model .Blood vessels are detected by thresholding methods and optic disc is detected by Hough transform [4]. The paper proposes on the detection of hard exudates. The advantage of the method is that it works well in low quality images also. Gaussian smoothening and contrast enhancement can be used as preprocessing steps [5]. Feature extraction based on location of optic disc, shape index and area is carried out for exudates detection. Wavelet sub bands can also be utilized for detecting the presence of exudates [6]. The energy distributions over the wavelet sub bands can be analyzed for exudates detection. The exudates are also detected effectively from the retina fundus image using segmentation algorithms [6].The paper is organized as follows. Section II covers the proposed method. Section III discusses the results obtained. Finally section IV deals with the conclusion.

\section{II.METHODOLOGY}

The flow chart of the proposed method is as follows. Image is taken from DIARETDB1 data base. The initial step is the blood vessel segmentation. The vessel regions are the intersecting regions between the high pass filtered image and the tophat reconstructed image. The optic disc is detected by using the circular Hough transform. After the detection of blood vessels and optic disc, these are removed. Therefore if there is any presence of exudates in the retina, these can be easily identified. Exudates are the leakages from the blood that gets deposited in the retinal areas. The exudates are detected by the imtool in the matlab 2011.

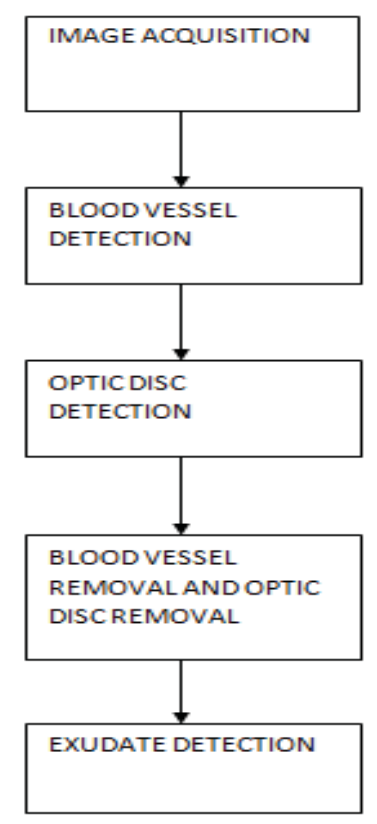

Fig 1.Flow chart of the proposed method

\section{A. Blood vessel segmentation}

The most important and initial step in the proposed method is the blood vessel segmentation. The images are collected from the DIARETDB1 and HRF databases. The green channel of the color fundus image is extracted. 
Contrast enhancement is performed to increase the visibility of the vessel regions. Contrast is the difference between the maximum and minimum pixel intensities. Contrast enhancement is done by histogram equalization. To make the vessel regions darker, vessel enhancement is performed. Vessel enhancement involves squaring the pixel values and normalizing in the range [0,1]. A low pass filter is implemented by means of median filtering. Median filtering is preferred since it reduces impulsive noises and preserves sharp edges. The vessel regions correspond to high frequencies in the images. Thus high pass filtered version of the image is obtained by subtracting the low pass image from the enhanced image. This yields the first preprocessed image. The red regions in the green plane image are extracted by taking the negative of the green plane image. Next, tophat reconstruction is performed on the negative image. Tophat reconstruction is a morphological reconstruction method that is defined as the difference between the input image and its opening by some structuring element. This is the second preprocessed image. The vessel regions are the intersected regions between the two preprocessed images.

\section{B. Optic disc detection}

Optic disc detection is performed by Hough transform. Hough transform is used to detect objects in an image. Circular Hough transform is an extension of Hough line transform. It is a feature extraction technique for detecting circles. A circle is represented by,

$$
(x-a)^{2}+(y-b)^{2}=r^{2}
$$

$(\mathrm{x}, \mathrm{y})$ is the center coordinate and $\mathrm{r}$ is the radius. The curve obtained in the Hough Transform space for each edge point will be a right circular cone. Point of intersection of the cones gives the parameters $a, b, r$. If the point (x, y) is fixed, then the parameters can be found according to (1). If the radius is fixed, the parameter space will correspond to be 2D. The main advantage of using Hough transform is that it is less affected by noise.

\section{C.Blood vessel and optic disc removal}

The blood vessels are detected by the intersecting regions between the high pass filtered image and tophat reconstructed image. The optic disc is detected by circular Hough transform. After the detection of these, the blood vessels and optic disc are subtracted from the original image. If there is the presence of exudates, then these are identified easily.

\section{Exudate detection.}

After the removal of blood vessels and optic disc, the exudates can be detected from the image by imtool in the matlab. The imtool facilitates the calculation of the pixel information. Based on the pixel information, the exudates are identified.

\section{III.RESULTS AND DISCUSSION}

The initial step in the Diabetic Retinopathy detection is the blood vessel segmentation. The vessel regions are the intersection regions between two preprocessed images. The first preprocessed image is the high pass filtered image and the second image is the tophat reconstructed image. High pass filtering is performed on the green channel and tophat reconstruction is performed on the red regions in the green channel. The results of blood vessel segmentation are given below: 


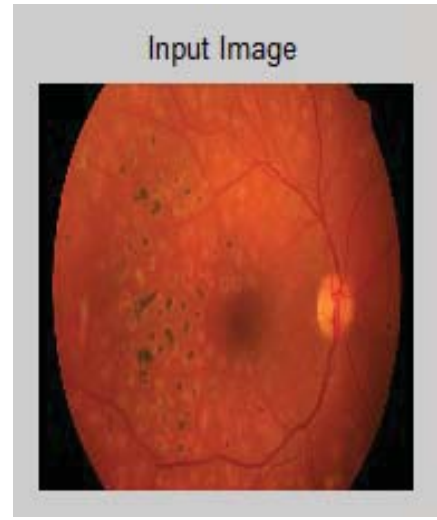

(a)

\section{Contrast Enhanced Image}

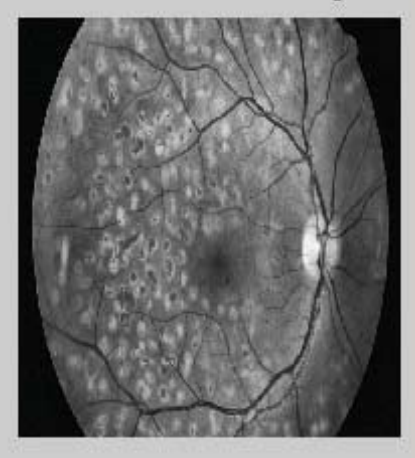

(c)

Binary image after highpass filtering

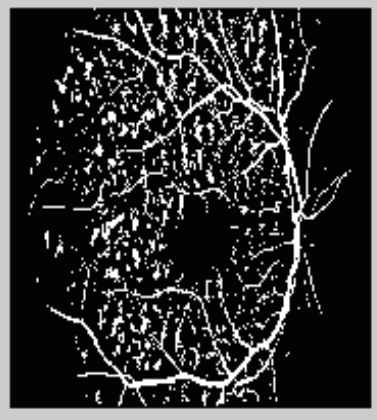

(e)

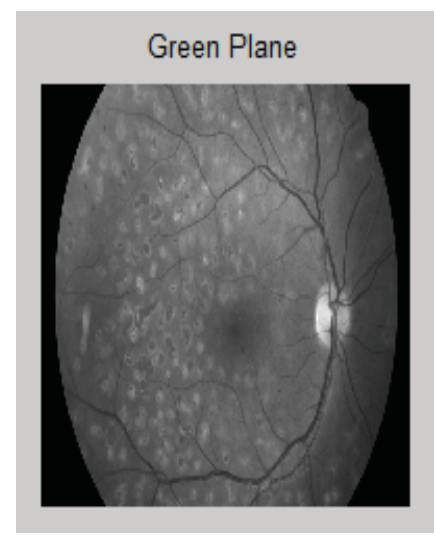

(b)

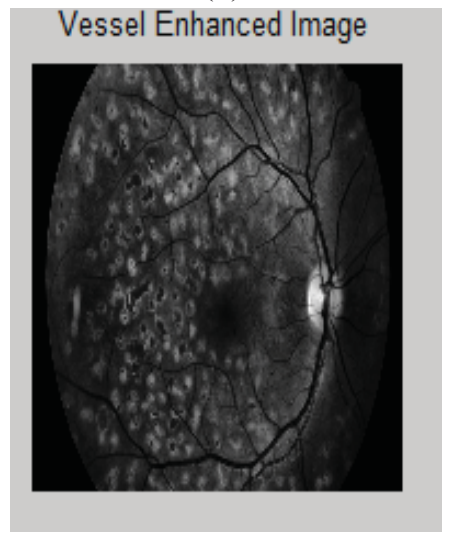

(d)

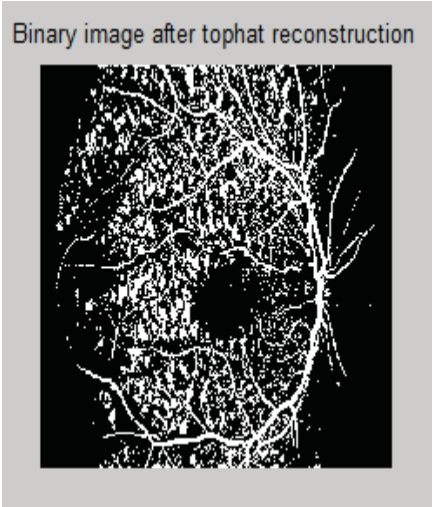

(f)

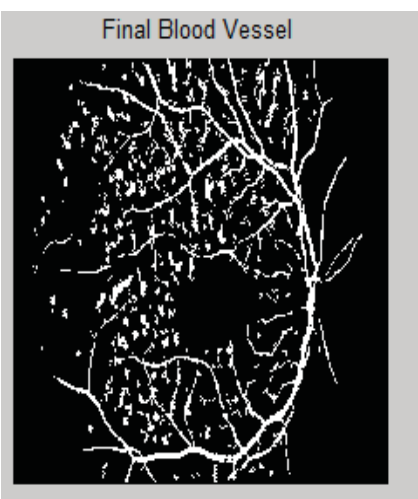


(g)

Fig 2. Results of blood vessel segmentation.(a) Input image (b) Green plane image (c) Contrast enhanced image (d) Vessel enhanced image (e) High pass filtered image (f) Tophat reconstructed image (g) Final vessel segmented image.

After the blood vessels are segmented from the fundus images, the optic disc is to be detected. Circular hough transform helps to detect circular objects in the image. The results of optic disc detection are given below.

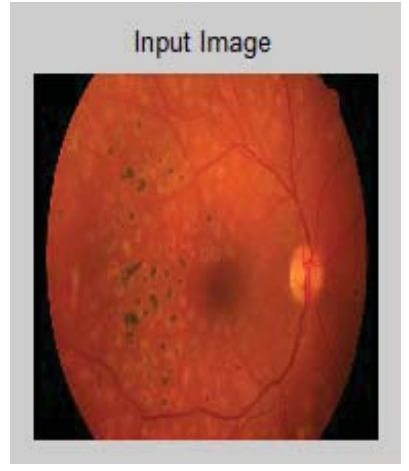

(a)

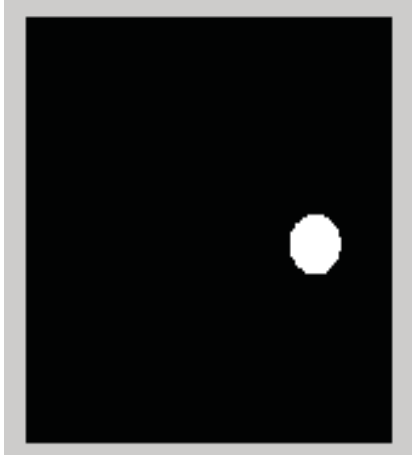

(b)

Fig 3. Optic disc segmentation result $\quad$ (a) Input image $\quad$ (b) Optic disc segmented image

Once the blood vessels and optic disc are detected these are subtracted from the input image. The results of blood vessel and optic disc removal are given below.

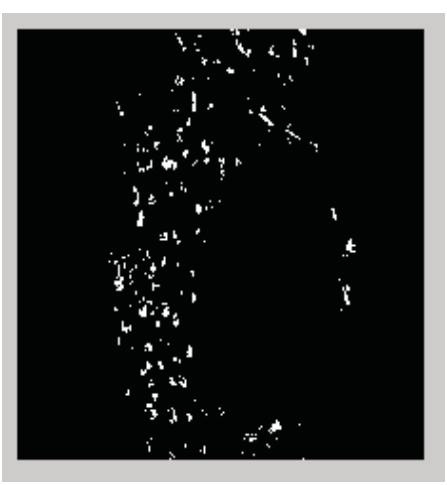

Fig 4. Results of blood vessel and optic disc removal

The exudates are detected by the imtool in the matlab. The imtool function makes use of the pixel information to determine whether it corresponds to exudates or not. The results of exudates detection is given below. 


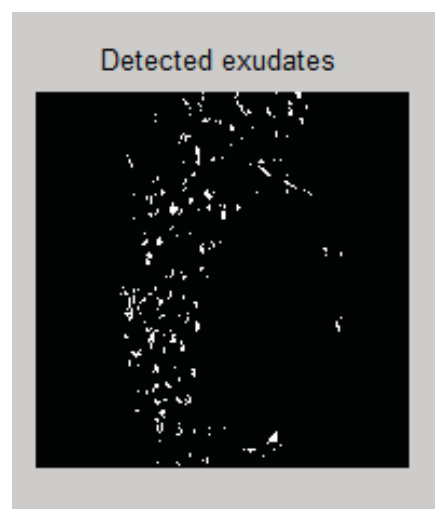

Fig 5 .Results of exudates detection

\section{IV.CONCLUSION}

The examination of blood vessels plays a major role in the medical field. Several approaches are available for the segmentation of blood vessels from the fundus images. The paper proposes a method that identifies vessel region as the intersected region between the high pass filtered image and tophat reconstructed image. Diabetic Retinopathy is a complicated disease that is caused by diabetes. The main symptom of Diabetic Retinopathy is the presence of exudates. The method in this paper makes use of the identification of exudates after the segmentation of blood vessels and optic disc. The exudates are detected by imtool operator. These methods provide a means to identify Diabetic Retinopathy to a certain extent.

\section{REFERENCES}

[1] D.Jeyashree,G.SharmilaandK.Ramasamy, "Combined Approach on Analysis of Retinal Blood Vessel Segmentation for Diabetic Retinopathy and Glaucoma Diagnosis", International Journal of Scientific \& Engineering Research, Volume 5, Issue 5, May-2014.

[2] R.Priya and P.Aruna, "Diagnosis of diabetic retinopathy using machine learning techniques", ICTACT Journal on Soft Computing, Volume 3, Issue 4, July 2013.

[3] Asha Gowda Karegowda, Asfiya Nasiha, M.A.Jayaram and A.S .Manjunath "Exudate detection in retinal images using back propagation neural network", International Journal of Computer Applications, Volume 25- No.3, July 2011.

[4] J.Ramya,S.Soundarya,A.Nagoormeeral, Rahmathnish and E.Revathi "Detection of exudates in color fundus images", International Journal of Innovative Research in Science,Engineering and Technology, Vol. 3, Issue 3, March 2014.

[5] Shraddha Tripathi, Krishna Kant Singh , B.K.Singh and Akansha Mehrotra, "Detection Automatic Detection of Exudates in Retinal Fundus Images using Differential Morphological Profile", International Journal of Engineering and Technology, Vol 5 No 3 Jun-Jul 2013

[6] Kullayamma and P. Madhavee Latha, "Retinal Image Analysis for Exudates Detection", International Journal of Engineering Research and Applications, Vol. 3, Issue 1, January -February 2013, pp.1871-1875. 